\title{
The English Galileo and His Vision of Projectile Motion under Air Resistance
}

\author{
Robert Kantrowitz $\mathbb{I D}^{1}$ and Michael M. Neumann $\mathbb{D}^{2}$ \\ ${ }^{1}$ Mathematics and Statistics Department, Hamilton College, 198 College Hill Road, Clinton, NY 13323, USA \\ ${ }^{2}$ Department of Mathematics and Statistics, Mississippi State University, Mississippi State, MS 39762, USA
}

Correspondence should be addressed to Robert Kantrowitz; rkantrow@hamilton.edu

Received 12 January 2020; Accepted 20 April 2020; Published 26 May 2020

Academic Editor: Hans Engler

Copyright ( $) 2020$ Robert Kantrowitz and Michael M. Neumann. This is an open access article distributed under the Creative Commons Attribution License, which permits unrestricted use, distribution, and reproduction in any medium, provided the original work is properly cited.

\begin{abstract}
This article presents a detailed discussion of the shape of the trajectory traced by a projectile under the forces of gravity and air resistance. In particular, our results confirm the insight of the English scientist Thomas Harriot into the motion of a projectile before the development of Newtonian classical mechanics. Our approach is based on the fact that the flight path of a resisted projectile is implemented by a strictly concave function for which the derivative is also strictly concave.
\end{abstract}

\section{Historical Background and the English Galileo}

The remarkable similarities between the scientific pursuits of Galileo Galilei (1564-1642) and his English contemporary, the mathematician and philosopher Thomas Harriot (1560-1621), led to the latter being dubbed "the English Galileo." Indeed, on the opening page of his monograph of that title, Schemmel [1] writes:

Harriot and Galileo were in fact occupied with very similar problems concerning astronomy, mechanics, optics, hydrodynamics, magnetism, and other fields of natural philosophy.

Though Galileo documented his work prolifically, Harriot published none of his results except for a small book on "the new found land of Virginia." Thus, while Galileo occupies a central position in the history of scientific developments, the name and the accomplishments of Harriot are far less established, and a systematic investigation of his nachlass comprising thousands of folio sheets has come into focus only recently. In addition to the extensive account [1] of Harriot and his work on projectile motion, the articles
$[2,3]$ also fill this historical gap by shedding light on the discoveries of the English Galileo.

Notable among their many and varied common undertakings is Galileo's and Harriot's study of an airborne projectile. In remarkable contrast to Galileo's idealized version in which gravity is the only active force, Harriot followed the practitioners' knowledge on projectile trajectories and tried to develop models that account for the effect of air resistance. Based on the practical experience of engineers and gunners at his time, Harriot envisioned that all realistic trajectories share an asymmetry that results from the air's influence, as depicted in Figure 1.

The curves shown in Figure 1 are based on the numerical solution of the differential equations resulting from Newton's law for projectile motion under air resistance quadratic in speed, as will be detailed in the last section. Although the modern approach is certainly far beyond the theoretical and computational tools available to preclassical mechanics, in his more advanced models, Harriot was able to construct ballistic trajectories that are astonishingly similar to those shown in Figure 1, see the folios shown in Figures 8 and 9 of [2], Figure 58 of [1], and Figures 4 and 5 of [3]. One of the specific aspects of the practitioners' knowledge that Harriot tried to address in 


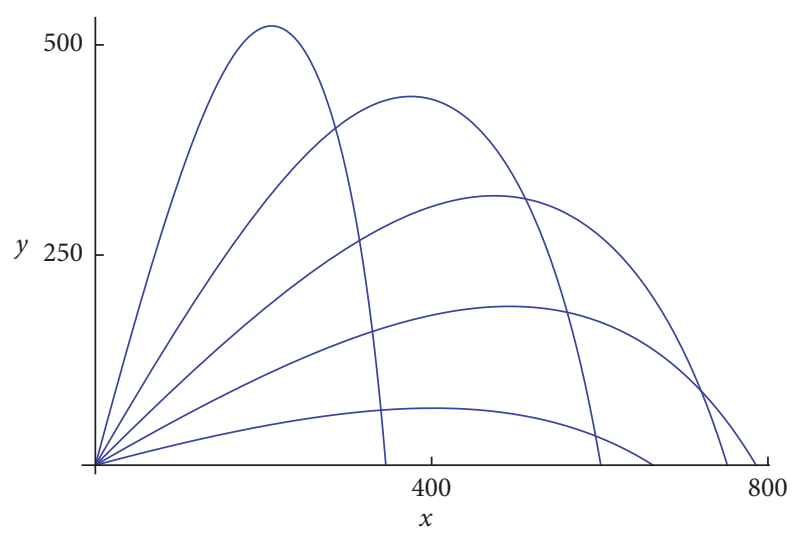

Figure 1: Flight trajectories under air resistance.

his models was the asymmetry of the trajectory in the following form.

The projectile's path from the muzzle of the gun to the culmination point of the trajectory is longer and flatter than the path from the culmination point down to the ground ([1], page 28).

The goal of this article is to confirm this observation by itemizing several comparisons between the ascending and the descending segments of a projectile's resisted flight trajectory. With this, we continue the line of inquiry that was recently developed in [4] for the special case of air resistance that is linear in speed. In this particular case, explicit formulas for the solutions of the relevant differential equations are available which allow the use of basic calculus to settle the issues at hand. Moreover, while no explicit solution formulas are known for the classical case of drag that is quadratic in speed, Hayen [5] was able to find a representation of the solutions in terms of certain quadratures that allowed him to obtain information about slope and arc length exactly as specified in assertions (i) and (vi) of our main result. We are indebted to one of the referees for providing us with reference [5].

There are, however, many other possibilities for air resistance, for instance, drag that is proportional to some power of the speed or drag that is dependent on the altitude of the projectile during flight. This means that a new approach is needed to handle the case of arbitrary air resistance.

In the next section, we frame the problem in an abstract, yet elementary setting that is quite apart from any specific application. It turns out that the strict concavity of a real-valued function together with that of its derivative determines certain geometric and analytic aspects of the graph of the function that are at the core of Harriot's vision.

In the final section, we return to the motivating ballistic application by describing a general framework for projectile motion in a plane under the influence of gravity and an opposing force of air resistance. Our comprehensive approach includes the cases of linear and quadratic drag, among many other models.

\section{Concave Functions and Main Result}

We recall that a real-valued function $g$ on an interval $J$ is said to be strictly concave provided that the estimate

$$
\operatorname{tg}(u)+(1-t) g(v)<g(t u+(1-t) v)
$$

holds for all distinct points $u, v \in J$ and all $t \in(0,1)$. It is well known that a differentiable function $g$ on $J$ is strictly concave precisely when $g^{\prime}$ is strictly decreasing on $J$, see, for instance, Theorem B of Section 1.12 of [6]. In particular, if $g$ is twice differentiable on $J$ and satisfies $g^{\prime \prime}(x)<0$ for all $x \in J$, then $g$ is strictly concave.

Throughout this section, we consider a continuously differentiable function $f$ on a closed bounded interval $[a, b]$ for which $f(a)=f(b)=0$ and suppose that both $f$ and $f^{\prime}$ are strictly concave. By the extreme value theorem, $f$ attains its maximum $m$ at some point $c \in[a, b]$, and the strict concavity of $f$ guarantees that this maximum point $c$ is unique and satisfies $a<c<b$ and $f(c)=m>0$. Moreover, by the standard characterization of strict concavity mentioned above, $f^{\prime}$ is strictly decreasing on $[a, b]$. Since Fermat's theorem ensures that $f^{\prime}(c)=0$, we conclude that $f^{\prime}>0$ on $[a, c)$ and $f^{\prime}<0$ on $(c, b]$. In particular, $f$ is strictly increasing on $[0, c]$ and strictly decreasing on $[c, b]$. Consequently, for each $h \in[0, m]$, there exist unique points $u \in[a, c]$ and $v \in[c, b]$ for which $f(u)=h=f(v)$, namely, $u=f_{1}^{-1}(h)$ and $v=f_{2}^{-1}(h)$, where $f_{1}$ and $f_{2}$ denote the restrictions of $f$ to $[a, c]$ and $[c, b]$, respectively.

As will be seen in the last section, for an arbitrary projectile that is shot from the ground level to the ground level and is subject to gravity and any kind of air resistance, the trajectory is implemented by a function $f$ for which both $f$ and $f^{\prime}$ are strictly concave. The concavity of this function and its derivative, together with the theorem proved in this section, implies that Figure 2 displays the typical shape of the trajectory traced by a projectile under air resistance and illustrates Harriot's vision.

We will verify, for instance, that the ascending branch is indeed strictly longer than the descending branch by establishing the inequality

$$
\int_{a}^{c} \sqrt{1+f^{\prime}(x)^{2}} \mathrm{~d} x>\int_{c}^{b} \sqrt{1+f^{\prime}(x)^{2}} \mathrm{~d} x .
$$

In the same vein, the area of the region under the ascending branch will be shown to be strictly larger than the corresponding area under the descending branch. As for flatness, we will establish Harriot's vision in a macroscopic or global sense by proving that the average value of the slope $f^{\prime}(x)$ on the ascending branch is strictly smaller than the average value of $-f^{\prime}(x)$ on the descending branch. Moreover, we will address a microscopic or pointwise version of Harriot's observation by showing that, in the setting of Figure 2, we have $f^{\prime}(u)<-f^{\prime}(v)$ whenever $a \leq u<c<v \leq b$ and $f(u)=f(v)$. All this is contained in the following main result of this article.

Theorem 1. Suppose that both $f$ and $f^{\prime}$ are strictly concave on $[a, b]$, and consider the setting of Figure 2. Then, for 


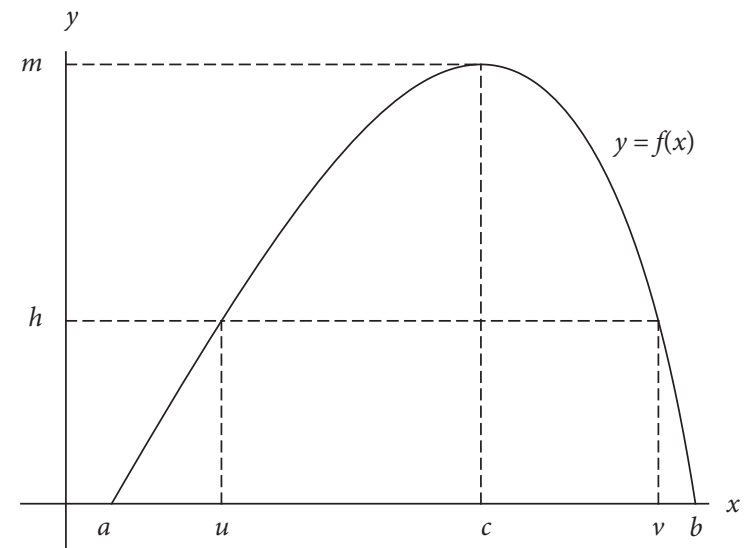

FIGURE 2: A strictly concave function with strictly concave derivative.

arbitrary points $u$ and $v$ for which $a \leq u<c<v \leq b$ and $f(u)=$ $f(v)=h$ for some $h \in[0, m)$, the following assertions hold:

(i) $f^{\prime}(u)<-f^{\prime}(v)$ and, in particular, $f^{\prime}(a)<-f^{\prime}(b)$;

(ii) $(u+v) / 2<c$ and, in particular, $(a+b) / 2<c$;

(iii) The average slopes satisfy

$$
\frac{1}{c-u} \int_{u}^{c} f^{\prime}(x) \mathrm{d} x<-\frac{1}{v-c} \int_{c}^{v} f^{\prime}(x) \mathrm{d} x
$$

(iv) The $x$-coordinate $d$ of the point of intersection of the tangent lines to $f$ at the points $(u, f(u))$ and $(v, f(v))$ satisfies $(u+v) / 2<d ;$

(v) The areas are related by

$$
\begin{gathered}
\int_{a}^{u} f(x) \mathrm{d} x>\int_{v}^{b} f(x) \mathrm{d} x, \\
\int_{u}^{c}(f(x)-h) \mathrm{d} x>\int_{c}^{v}(f(x)-h) \mathrm{d} x ;
\end{gathered}
$$

in particular, we have

$$
\int_{a}^{c} f(x) \mathrm{d} x>\int_{c}^{b} f(x) \mathrm{d} x
$$

(vi) The arc lengths satisfy the estimates

$$
\begin{aligned}
& \int_{a}^{u} \sqrt{1+f^{\prime}(x)^{2}} \mathrm{~d} x>\int_{v}^{b} \sqrt{1+f^{\prime}(x)^{2}} \mathrm{~d} x, \\
& \int_{u}^{c} \sqrt{1+f^{\prime}(x)^{2}} \mathrm{~d} x>\int_{c}^{v} \sqrt{1+f^{\prime}(x)^{2}} \mathrm{~d} x
\end{aligned}
$$

in particular, we have

$$
\int_{a}^{c} \sqrt{1+f^{\prime}(x)^{2}} \mathrm{~d} x>\int_{c}^{b} \sqrt{1+f^{\prime}(x)^{2}} \mathrm{~d} x .
$$

Proof.

(i) By the strict concavity of $f^{\prime}$, we have

$$
t f^{\prime}(u)+(1-t) f^{\prime}(v)<f^{\prime}(t u+(1-t) v),
$$

for all $t \in(0,1)$ and therefore

$\int_{0}^{1}\left[t f^{\prime}(u)+(1-t) f^{\prime}(v)\right] \mathrm{d} t<\int_{0}^{1} f^{\prime}(t u+(1-t) v) \mathrm{d} t$.

On the left-hand side, it is trivial to perform the integration, while the integral on the right-hand side may be handled with the substitution $x=t u+(1-t) v$. It follows that

$$
\frac{f^{\prime}(u)+f^{\prime}(v)}{2}<\frac{1}{u-v} \int_{v}^{u} f^{\prime}(x) \mathrm{d} x=\frac{f(u)-f(v)}{u-v} .
$$

Hence, the condition $f(u)=f(v)$ entails the desired estimate $f^{\prime}(u)<-f^{\prime}(v)$.

(ii) We know that the two restrictions $f_{1}=f \mid[a, c]$ and $f_{2}=f \mid[c, b]$ are injective with range $[0, m]$. Moreover, we have $f_{1}^{\prime}>0$ on $[a, c)$ and $f_{2}^{\prime}<0$ on $(c, b]$. This ensures that the inverses of $f_{1}$ and $f_{2}$ are differentiable on $[0, m)$ and satisfy

$$
\begin{aligned}
& \left(f_{1}^{-1}\right)^{\prime}(y)=\frac{1}{f^{\prime}\left(f_{1}^{-1}(y)\right)}>0, \\
& \left(f_{2}^{-1}\right)^{\prime}(y)=\frac{1}{f^{\prime}\left(f_{2}^{-1}(y)\right)}<0,
\end{aligned}
$$

for all $y \in[0, m)$. Since assertion (i) guarantees that $f^{\prime}\left(f_{1}^{-1}(y)\right)<-f^{\prime}\left(f_{2}^{-1}(y)\right)$, we obtain

$$
\left(f_{1}^{-1}\right)^{\prime}(y)=\frac{1}{f^{\prime}\left(f_{1}^{-1}(y)\right)}>-\frac{1}{f^{\prime}\left(f_{2}^{-1}(y)\right)}=-\left(f_{2}^{-1}\right)^{\prime}(y)
$$

and therefore $\left(f_{1}^{-1}\right)^{\prime}(y)+\left(f_{2}^{-1}\right)^{\prime}(y)>0$ for all $y \in[0, m)$.

Now, given arbitrary points $\alpha$ and $\beta$ with $0 \leq \alpha<\beta<m$, integration leads to

$$
\int_{\alpha}^{\beta}\left(\left(f_{1}^{-1}\right)^{\prime}(y)+\left(f_{2}^{-1}\right)^{\prime}(y)\right) \mathrm{d} y>0
$$

and hence $f_{1}^{-1}(\alpha)+f_{2}^{-1}(\alpha)<f_{1}^{-1}(\beta)+f_{2}^{-1}(\beta)$. This shows that the function $f_{1}^{-1}+f_{2}^{-1}$ is strictly increasing on $[0, m)$ and, since this function is continuous, also on $[0, m]$. In particular, we obtain $f_{1}^{-1}(\alpha)+f_{2}^{-1}(\alpha)<f_{1}^{-1}(m)+f_{2}^{-1}(m)=2 c \quad$ whenever $0 \leq \alpha<m$. The choice $\alpha=h=f(u)=f(v)$ then yields $u+v<2 c$, the desired estimate.

(iii) Because $h=f(u)=f(v)$, the stipulated estimate for the average values is equivalent to 


$$
\frac{f(c)-h}{c-u}<-\frac{h-f(c)}{v-c} .
$$

But this means precisely that $(u+v) / 2<c$ which was established in part (ii).

(iv) Since $f$ is strictly concave, we know that $f^{\prime}$ is strictly decreasing. Thus, $f^{\prime}(u)>f^{\prime}(v)$ and therefore $f^{\prime}(u)-f^{\prime}(v)>0$. This ensures that the two tangent lines in question have a unique point of intersection. Indeed, since these lines are given by the formulas

$$
\begin{aligned}
& \ell_{u}(x)=f(u)+f^{\prime}(u)(x-u), \\
& \ell_{v}(x)=f(v)+f^{\prime}(v)(x-v),
\end{aligned}
$$

for all $x \in \mathbb{R}$, the equation $\ell_{u}(d)=\ell_{v}(d)$ has the unique solution:

$$
d=\frac{f(v)-f(u)+u f^{\prime}(u)-v f^{\prime}(v)}{f^{\prime}(u)-f^{\prime}(v)} .
$$

Simple algebra then reveals that the inequality $(u+$ $v) / 2<d$ may be rewritten in the following form:

$$
\frac{f^{\prime}(u)+f^{\prime}(v)}{2}<\frac{f(u)-f(v)}{u-v},
$$

and thus holds by the strict concavity of $f^{\prime}$, as shown in the proof of assertion (i). Note that the condition $f(u)=f(v)$ is not essential here and that the preceding estimate for arbitrary distinct points $u, v$ in $[a, b]$ actually characterizes the strict concavity of $f^{\prime}$ on $[a, b]$, see Corollary 1 of [7].

(v) To establish the first inequality, we recall from the proof of part (ii) that

$$
\left(f_{1}^{-1}\right)^{\prime}(y)>-\left(f_{2}^{-1}\right)^{\prime}(y), \quad \text { for all } y \in[0, m) .
$$

Hence, the substitutions $x=f_{1}^{-1}(y)$ and $x=f_{2}^{-1}(y)$ lead to

$$
\begin{gathered}
\int_{a}^{u} f(x) \mathrm{d} x=\int_{0}^{h} y\left(f_{1}^{-1}\right)^{\prime}(y) \mathrm{d} y \\
>-\int_{0}^{h} y\left(f_{2}^{-1}\right)^{\prime}(y) \mathrm{d} y=\int_{h}^{0} y\left(f_{2}^{-1}\right)^{\prime}(y) \mathrm{d} y=\int_{v}^{b} f(x) \mathrm{d} x,
\end{gathered}
$$

which settles the first claim. For the proof of the second inequality, we observe that, for arbitrary $y \in[0, m)$, the estimate $f_{1}^{-1}(y)+f_{2}^{-1}(y)<2 c$ from the proof of assertion (ii) may be rewritten in the form $c-f_{1}^{-1}(y)>f_{2}^{-1}(y)-c$. Switching to integration with horizontal cross-sections, we then obtain

$$
\begin{aligned}
\int_{u}^{c}(f(x)-h) \mathrm{d} x & =\int_{h}^{m}\left(c-f_{1}^{-1}(y)\right) \mathrm{d} y>\int_{h}^{m}\left(f_{2}^{-1}(y)-c\right) \mathrm{d} y \\
& =\int_{c}^{v}(f(x)-h) \mathrm{d} x,
\end{aligned}
$$

as desired. Moreover, since we know from part (ii) that $(u+v) / 2<c$ and hence $h(c-u)>h(v-c)$, we also arrive at the estimate

$$
\int_{u}^{c} f(x) \mathrm{d} x>\int_{c}^{v} f(x) \mathrm{d} x
$$

The final claim is immediate from this and the first inequality. Alternatively, the final claim follows from the second inequality in the special case $h=0$.

(vi) To establish the first claim, we define the function $\varphi$ by $\varphi(z)=f_{2}^{-1}\left(f_{1}(z)\right)$, where, as above, $f_{1}$ and $f_{2}$ stand for the restrictions of $f$ to $[a, c]$ and $[c, b]$, respectively. Obviously, $\varphi(a)=b, \varphi(u)=v$, and $\varphi$ maps $[a, u]$ onto $[v, b]$. Moreover, because $\varphi=f_{2}^{-1} \circ f_{1}$ on the interval $[a, u]$, the chain rule confirms that $\varphi$ is differentiable on $[a, u]$. Noting that $f(\varphi(z))=f_{2}(\varphi(z))=f_{1}(z)=f(z)$, we arrive at $f^{\prime}(\varphi(z)) \varphi^{\prime}(z)=f^{\prime}(z)$ and therefore

$$
\varphi^{\prime}(z)=\frac{f^{\prime}(z)}{f^{\prime}(\varphi(z))}<0,
$$

for all $z \in[a, u]$. Since assertion (i) ensures that $f(z)<-f^{\prime}(\varphi(z))$, we conclude that $\left|\varphi^{\prime}(z)\right|<1$ for all $z \in[a, u]$. We now employ the substitution $x=\varphi(z)$ to obtain

$$
\begin{aligned}
\int_{v}^{b} \sqrt{1+f^{\prime}(x)^{2}} \mathrm{~d} x & =\int_{u}^{a} \sqrt{1+f^{\prime}(\varphi(z))^{2}} \varphi^{\prime}(z) \mathrm{d} z \\
& =\int_{a}^{u} \sqrt{1+f^{\prime}(\varphi(z))^{2}}\left|\varphi^{\prime}(z)\right| \mathrm{d} z \\
& =\int_{a}^{u} \sqrt{\varphi^{\prime}(z)^{2}+f^{\prime}(\varphi(z))^{2} \varphi^{\prime}(z)^{2}} \mathrm{~d} z \\
& =\int_{a}^{u} \sqrt{\varphi^{\prime}(z)^{2}+f^{\prime}(z)^{2}} \mathrm{~d} z,
\end{aligned}
$$

where we again used the fact that $f^{\prime}(\varphi(z)) \varphi^{\prime}(z)=f^{\prime}(z)$. Because $\left|\varphi^{\prime}(z)\right|<1$ for all $z \in[a, u]$, we arrive at

$$
\int_{v}^{b} \sqrt{1+f^{\prime}(x)^{2}} \mathrm{~d} x<\int_{a}^{u} \sqrt{1+f^{\prime}(z)^{2}} \mathrm{~d} z,
$$

which establishes the first claim. Taking the limit as $h$ approaches $m$, both $u$ and $v$ tend to $c$, which results in

$$
\int_{c}^{b} \sqrt{1+f^{\prime}(x)^{2}} \mathrm{~d} x \leq \int_{a}^{c} \sqrt{1+f^{\prime}(z)^{2}} \mathrm{~d} z .
$$


Since this holds for an arbitrary base interval $[a, b]$, it also holds for every subinterval $[u, v]$ for which $f(u)=f(v)$. Hence,

$$
\int_{c}^{v} \sqrt{1+f^{\prime}(x)^{2}} \mathrm{~d} x \leq \int_{u}^{c} \sqrt{1+f^{\prime}(z)^{2}} \mathrm{~d} z .
$$

Together with (24) we obtain, in fact, the strict inequality:

$$
\int_{c}^{b} \sqrt{1+f^{\prime}(x)^{2}} \mathrm{~d} x<\int_{a}^{c} \sqrt{1+f^{\prime}(z)^{2}} \mathrm{~d} z .
$$

Here, again, we may replace the interval $[a, b]$ by any subinterval $[u, v]$ for which $f(u)=f(v)$. This establishes the remaining claim.

\section{Connection to Ballistics}

In this section, we consider the typical motion of a projectile in the $x y$-plane. We suppose that the projectile is launched at time $t=0$ from the origin with muzzle speed $s>0$ and angle of inclination $\theta \in(-\pi / 2, \pi / 2)$ relative to the positive $x$-axis. The position vector of the projectile at time $t \geq 0$ is denoted by $\mathbf{r}(t)=\langle x(t), y(t)\rangle$.

As usual, we suppose that the motion of the projectile is governed by two forces. One of these forces is gravity in the direction of the negative $y$-axis which results in acceleration of a given magnitude $g>0$. The other force is air resistance whose direction is opposite to the velocity vector $\mathbf{r}^{\prime}(t)=$ $\left\langle x^{\prime}(t), y^{\prime}(t)\right\rangle$ of the projectile and whose magnitude is proportional to the mass $m$ of the projectile. Specifically, as in [7], the retarding force is supposed to be represented by

$$
-m W\left(t, x(t), x^{\prime}(t), y(t), y^{\prime}(t)\right)\left\langle x^{\prime}(t), y^{\prime}(t)\right\rangle,
$$

where $W$ is a given non-negative continuous function of five variables, defined on a suitable domain in $\mathbb{R}^{5}$. In addition to the case $W=0$ of no air resistance, this model covers the important case where the drag is proportional to some power of the speed

$$
\left|\mathbf{r}^{\prime}(t)\right|=\sqrt{x^{\prime}(t)^{2}+y^{\prime}(t)^{2}},
$$

of the projectile, see Chapter 3 of [8].

By Newton's law, the projectile motion is now described by the initial value problem:

$$
\begin{aligned}
& x^{\prime \prime}(t)=-W\left(t, x(t), x^{\prime}(t), y(t), y^{\prime}(t)\right) x^{\prime}(t), \\
& y^{\prime \prime}(t)=-g-W\left(t, x(t), x^{\prime}(t), y(t), y^{\prime}(t)\right) y^{\prime}(t), \\
& x(0)=0, \\
& y(0)=0, \\
& x^{\prime}(0)=s \cos (\theta), \\
& y^{\prime}(0)=s \sin (\theta) .
\end{aligned}
$$

As in [7], we assume that the drag function $W$ is admissible in the sense that, for every choice of $s>0$ and $-\pi / 2<\theta<\pi / 2$, the initial value problem (30) has a unique solution $(x(t), y(t))$ that is defined for all $t \geq 0$. In practice, admissibility is guaranteed by existence and uniqueness results of the Picard-Lindelöf type under mild Lipschitz conditions on the function $W$ with respect to the last four variables, see, for instance, [9].

The graphics in Figure 1 displays the numerical solution of (30) generated by the NDSOLVE command of Mathematica for the particular choices

$$
W\left(t, x(t), x^{\prime}(t), y(t), y^{\prime}(t)\right)=0.002 \sqrt{x^{\prime}(t)^{2}+y(t)^{2}},
$$

$g=9.81 \mathrm{~m} / \mathrm{sec}^{2}, s=200 \mathrm{~m} / \mathrm{sec}$, and the five canonical values of the launch angle $\theta=15^{\circ}, 30^{\circ}, 45^{\circ}, 60^{\circ}$, and $75^{\circ}$.

In general, explicit formulas for the solution $(x(t), y(t))$ of (30) are not available. In fact, even in the special case of drag quadratic in speed, no such formulas are known, see $[8,10]$, and [11]. Nevertheless, as shown in Section 4 of [7], it is possible to obtain geometric insight into the shape of the trajectory traced by the projectile. First, it turns out that the function $x$ is strictly increasing on $[0, \infty)$ and that its range is an interval of the form $\left[0, x_{\infty}\right)$ where $0<x_{\infty} \leq \infty$. Furthermore, by Theorem 12 of [7], we have

Theorem 2. Suppose that $W$ is a strictly positive admissible dragfunction, and let $(x(t), y(t))$ for $t \geq 0$ denote the solution of the corresponding initial value problem (30). Then, this solution is implemented by the function $f=y \circ x^{-1}$ on the range $\left[0, x_{\infty}\right)$ of $x$ in the sense that $f(x(t))=y(t)$ for all $t \geq 0$. Moreover, $f$ is three times differentiable, and both $f$ and $f^{\prime}$ are strictly concave on $\left[0, x_{\infty}\right)$.

Indeed, the elementary proof boils down to the fact that

$$
\begin{aligned}
f^{\prime \prime}(x(t)) & =-\frac{g}{x^{\prime}(t)^{2}}<0, \\
f^{\prime \prime \prime}(x(t)) & =\frac{2 g x^{\prime \prime}(t)}{x^{\prime}(t)^{4}} \\
& =-\frac{2 g W\left(t, x(t), x^{\prime}(t), y(t), y^{\prime}(t)\right)}{x^{\prime}(t)^{3}}<0,
\end{aligned}
$$

for all $t \geq 0$. Harriot's vision is now established in remarkable generality by Theorems 1 and 2 .

\section{Data Availability}

No data were used to support this study.

\section{Conflicts of Interest}

The authors declare that they have no conflicts of interest.

\section{References}

[1] M. Schemmel, The English Galileo: Thomas Harriot's Work on Motion as an Example of Preclassical Mechanics, Springer, Berlin, Germany, 2008.

[2] M. Schemmel, "The English Galileo: Thomas Harriot and the force of shared knowledge in early modern mechanics," Physics in Perspective, vol. 8, no. 4, pp. 360-380, 2006. 
[3] R. Smith, "Shining a light on Harriot and Galileo: on the mechanics of reflection and projectile motion," History of Science, vol. 53, no. 3, pp. 296-319, 2015.

[4] C. W. Groetsch, "Harriot's observation of resisted trajectories," The Citadel, Charleston, SC, USA, 2018.

[5] J. C. Hayen, "Projectile motion in a resistant medium (part I: exact solution and properties)," International Journal of NonLinear Mechanics, vol. 38, no. 3, pp. 357-369, 2003.

[6] A. W. Roberts and D. E. Varberg, Convex Functions, Academic Press, New York, NY, USA, 1973.

[7] R. Kantrowitz and M. M. Neumann, "Parabolic sandwiches for functions on a compact interval and an application to projectile motion," International Journal of Mathematics and Mathematical Sciences, vol. 2019, Article ID 4868106, 7 pages, 2019.

[8] N. de Mestre, The Mathematics of Projectiles in Sport, Cambridge University Press, Cambridge, UK, 1990.

[9] E. A. Coddington and N. Levinson, Theory of Ordinary Differential Equations, McGraw-Hill, New York, NY, USA, 1955.

[10] W. W. Hackborn, "Motion through air: what a drag," $\mathrm{Ca}$ nadian Applied Mathematics Quarterly, vol. 14, pp. 285-298, 2006.

[11] W. W. Hackborn, "Projectile motion: resistance is fertile," The American Mathematical Monthly, vol. 115, no. 9, pp. 813-819, 2008. 